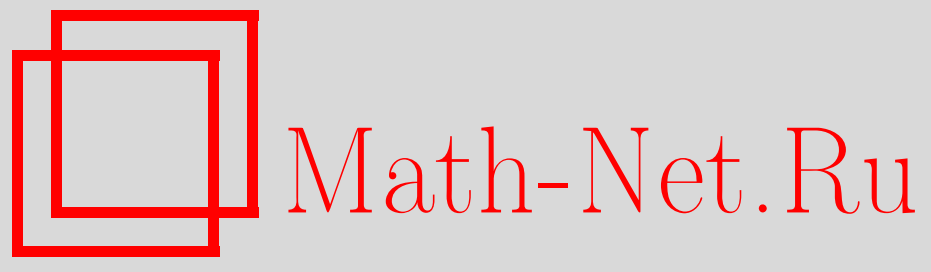

С. А. Белев, Н. А. Тюрин, Подъемы лагранжевых торов, матем. заметки, 2012, том 91, выпуск 5, 784-786

DOI: https://doi.org/10.4213/mzm9363

Использование Общероссийского математического портала Math-Net.Ru подразумевает, что вы прочитали и согласны с пользовательским соглашением http://www . mathnet.ru/rus/agreement

Параметры загрузки:

IP : 52.205 .19 .152

26 апреля 2023 г., 17:48:50

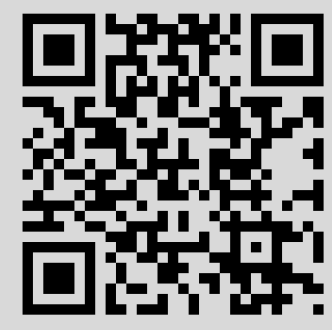




\section{Подъемы лагранжевых торов}

\section{С. А. Белёв, Н. А. Тюрин}

В настоящей работе мы продолжаем исследования лагранжевой геометрии компактных симплектических многообразий, обладающих псевдоторической структурой. В отличие от [1], где строились лагранжевы слоения с гладким общим слоем, ниже мы занимаемся задачей построения лагранжевых торов.

Напомним, псевдоторической структурой на компактном симплектическом многообразии $\left(X, \omega_{X}\right)$ вещественной размерности $2 n$ называется набор данных $\left(f_{1}, \ldots, f_{k}, B, \psi, Y\right)$, состоящий из набора гладких морсовских функций $f_{i}$, алгебраически не зависимых почти всюду на $X$ и попарно коммутирующих, и семейства симплектических подмногообразий $\left\{Q_{p}=\overline{\psi^{-1}(p)}=\psi^{-1}(p) \cup B, p \in Y\right\}$ вещественной размерности $2 k$ с базисным множеством $B \subset X$, параметризуемого торическим симплектическим многообразием $\left(Y, \omega_{Y}\right)$ вещественной размерности $2(n-k)$, так что выполнены следующие обобщенные коммутационные соотношения. Во-первых, гамильтонов поток, индуцируемый каждой $f_{i}$, сохраняет каждый элемент семейства $Q_{p}$; во-вторых, для любой гладкой функции $h \in C^{\infty}(Y, \mathbb{R})$ поднятое посредством симплектической связности $\nabla_{\psi}$ гамильтоново векторное поле $X_{h}$ коллинеарно гамильтонову векторному полю поднятой функции $\psi^{*} h$ всюду на $X \backslash B$, т.е.

$$
\nabla_{\psi} X_{h} \wedge X_{\psi^{*} h} \equiv 0
$$

для любой $h \in C^{\infty}(Y, \mathbb{R})$ (детали определения см. [2]). Слой $Q_{p}$, гладкий вне базисного множества $B$, мы называем регулярным. Псевдоторическую структуру $\left(f_{1}, \ldots, f_{k}, B, \psi, Y\right)$ с регулярным общим слоем мы называем регулярной. Для регулярной псевдоторической структуры множество точек $p \in Y$, параметризующих нерегулярные слои, составляет подмногообразие $D_{\operatorname{Sing}} \subset Y$. Отображение вещественными интегралами $F=\left(f_{1}, \ldots, f_{k}\right)$ : $X \rightarrow \mathbb{R}^{k}$ определяет выпуклый многогранник $P_{Q} \subset \mathbb{R}^{k}$, изоморфный многограннику моментов торического многообразия $\left(Q_{p},\left(f_{1}, \ldots, f_{k}\right)\right)$ для общего $p \in Y$, так что $F(B)=\partial P_{Q}$; особенности нерегулярных слоев $\operatorname{Sing}_{\psi} \subset X \backslash B$ составляют подмножество в детерминантах $\Delta\left(f_{1}, \ldots, f_{k}\right)=\left\{d f_{1} \wedge \ldots d f_{k}=0\right\} \subset X$, причем во всех известных на сегодняшний день примерах $\Delta\left(f_{1}, \ldots, f_{k}\right)=B \cup \operatorname{Sing}_{\psi}$. Образ $F\left(\operatorname{Sing}_{\psi}\right)=P_{\operatorname{Sing}} \subset P_{Q}$ есть подмножество коразмерности 1 в многограннике $P_{Q}$, состоящее из пересечений $P_{Q}$ с некоторыми гиперплоскостями $\pi_{1}, \ldots, \pi_{m} \subset \mathbb{R}^{k}$. При этом нерегулярный слой $Q_{p}, p \in D_{\text {Sing }}$ есть приводимое многообразие, каждая компонента которого сама по себе является торическим многообразием, многранник моментов которого получается отрезанием поднабором гиперплоскостей $\pi_{i}$ соответствующей части от $P_{Q}$. Таким образом, для регулярного слоя $Q_{p}$ прообразом внутренней точки $F^{-1}(x) \cap Q_{p}, x \in P_{Q} \backslash \partial P_{Q}$, будет гладкий лагранжев $k$-мерный тор; для нерегулярного слоя $Q_{p}$ прообразом точки $F^{-1}(x) \cap Q_{p}, x \in P_{Q} \backslash\left(\partial P_{Q} \cup P_{\text {Sing }}\right)$, снова будет гладкий $k$-мерный тор, но если $x \in P_{\text {Sing }}$, то прообразом будет сингулярный тор специального вида (подробности в [1], [2]).

Пример. Реализуем многообразие флагов $F^{3}$ как гиперповерхность в прямом произведении $\mathbb{C P}^{2} \times \mathbb{C P}^{2}$, задаваемую уравнением $x_{0} y_{0}+x_{1} y_{1}+x_{2} y_{2}=0$; тогда имеется псевдоторическая структура $\left(f_{1}, f_{2}, B, \psi, Y\right)$, где $B \subset F^{3}$ - шестиугольник, состоящий из шести проективных прямых, $Y$ есть $\mathbb{C P}^{1}$, а $D_{\text {Sing }} \subset Y$ состоит из трех точек. Далее, в качестве $P_{Q} \subset \mathbb{R}^{2}$ имеем плоский выпуклый шестиугольник, являющийся многоугольником моментов торической поверхности дель Пеццо $\mathbb{C P}_{3}^{2}$, а $P_{\text {Sing }} \subset P_{Q}$ состоит из трех диагоналей $P_{Q}$, соединяющих попарно противоположные вершины. Каждая такая диагональ делит $P_{Q}$ на два четырехугольника, каждый из которых является выпуклым многогранником для

Работа выполнена при поддержке совместного гранта Российского фонда фундаментальных исследований (№ 11-01-00980-а) и НРУ ВШЭ (№ 11-09-0038), а также при поддержке Лаборатории алгебраической геометрии ГУ-ВШЭ (грант правительства РФ дог. 11.G34.31.0023). 
торической поверхности дель Пеццо $\mathbb{C P}_{1}^{2}$, и каждый из трех особых слоев $Q_{p}, p \in D_{\operatorname{Sing}}$ есть пара таких поверхностей дель Пеццо, пересекающихся по прямой - прообразу соответствующей диагонали. Таким образом, множество особенностей Sing есть три такие прямые (детали этой конструкции см. в [3]).

Основное наблюдение настоящей работы содержит следующая

Теорема. Пусть на компактном симплектическом многообразии $\left(X, \omega_{X}\right)$ задана регулярная псевдоторическая структура $\left(f_{1}, \ldots, f_{k}, B, \psi, Y\right)$. Тогда

1) для произвольного гладкого лагранжева тора $T \subset Y$ и произвольного набора значений $\left(c_{1}, \ldots, c_{k}\right)=x \in \mathbb{R}^{k}$ вещественных интегралов $\left(f_{1}, \ldots, f_{k}\right)$ из области $x \in$ $P_{Q} \backslash\left(\partial P_{Q} \cup P_{\text {Sing }}\right)$ существует подвем $T$ до гладкого лагранжева тора $T(x) \subset X$;

2) для произвольного гладкого лагранжева тора $T \subset Y$ такого, ито $T \cap D_{\text {Sing }}=\varnothing$, и произвольного набора значений $\left(c_{1}, \ldots, c_{k}\right)=x \in \mathbb{R}^{k}$ вещественных интегралов $\left(f_{1}, \ldots, f_{k}\right)$ из области $x \in P_{Q} \backslash \partial P_{Q}$ существует подвем $T$ до гладкого лагранжева mopa $T(x) \subset X$.

Случай, когда $T \subset Y$ есть стандартный тор в симплектическом торическом многообразии $Y$, следует из доказательства предложения 2 [1]. Для произвольного тора модифицируем основную идею конструкции из [1], а именно, построим локальный набор функций $\left(h_{1}, \ldots, h_{n-k}\right)$ на $Y$ вблизи $T$ таких, что $\left\{h_{i}, h_{j}\right\}_{\omega_{Y}}=0$ и $T$ есть совместное множество уровня этих функций, соответствующее некритическим значениям.

Итак, пусть $T \subset Y$, а $\left(c_{1}, \ldots, c_{k}\right)=x \in \mathbb{R}^{k}$ набор значений $f_{1}, \ldots, f_{k}$ как в условиях 1$)$ или 2). Тогда для каждого слоя $Q_{p} \subset X$ над точкой $p \in T \subset Y$ выбор значений функций определяет гладкий лагранжев тор $S_{c_{1}, \ldots, c_{k}}^{p}$. Объединяя эти торы вдоль $T \subset Y$, получаем

$$
T(x)=\bigcup_{p \in T} S_{c_{1}, \ldots, c_{k}}^{p},
$$

обладающий структурой расслоения над $(n-k)$-мерным тором со слоем $k$-мерный тор. Покажем, что $T(X)$ является лагранжевым тором. Для этого достаточно построить на $T(X)$ набор векторных полей $\nu_{1}, \ldots, \nu_{n}$, глобально тривиализующих касательное расслоение $T T(x)$ и удовлетворяющих условию $\omega_{X}\left(\nu_{i}, \nu_{j}\right) \equiv 0$ на $T(x)$.

Набор $\nu_{1}, \ldots, \nu_{n}$ частично уже существует на $T(x)$, так как всякая функция из набора $f_{1}, \ldots, f_{k}$ гамильтоновым действием сохраняет каждое из $S_{c_{1}, \ldots, c_{k}}^{p}$, т.е.

$$
\nu_{i}=\left.X_{f_{i}}\right|_{T(x)}, \quad i=1, \ldots, k .
$$

Построим теперь недостающие векторные поля. Для этого покажем, что для любого лагранжева тора $T \subset Y$ найдется локальный набор первых интегралов $h_{1}, \ldots, h_{n-k}$, чьи гамильтоновы векторные поля сохраняют $T$. В самом деле, согласно теореме Дарбу-Вейнстейна [4] любое лагранжево подмногообразие $S \subset Y$ обладает окрестностью $N(S) \subset Y$, симплектоморфной некоторой малой окрестности $N_{0}(S) \subset T^{*} S$ нулевого сечения кокасательного расслоения. Так как в нашем случае $S=T$ есть тор, взяв на $T^{*} T$ набор коммутирующих функций $p_{1}, \ldots, p_{n-k}$, соотвествующих координатам “действия", обрезав все $p_{i}$ так, чтобы они занулились на границе $N_{0}(S)$, и затем перенося результат на $N(S)$, мы получим необходимый локальный набор $h_{1}, \ldots, h_{n-k}$. Теперь воспользуемся подъемом $\psi^{*}: C^{\infty}(Y, \mathbb{R}) \rightarrow C^{\infty}(X \backslash(B \cup \operatorname{Sing}))$ гладких функций и определим локальные гладкие функции $\psi^{*} h_{1}, \ldots, \psi^{*} h_{n-k}$ вблизи $T(x) \subset X$. При этом заметим, что условия 1$)$ и 2$)$ удостоверяют в том, что в обоих этих случаях $T(x)$ не пересекается с $B \cup \operatorname{Sing}$ и, значит, обладает некоторой окрестностью, для которой работает подъем $\psi^{*}$. По определению псевдоторической структуры $\nabla_{\psi} X_{h_{i}}$ коллинеарен $X_{\psi^{*} h_{i}}$ откуда следуют коммутационные соотношения для $f_{i}$ и $\psi^{*} h_{j}$, и значит в качестве $\nu_{j}$ при $j>k$ можно взять $\nu_{j}=\left.X_{\psi^{*} h_{j-k}}\right|_{T(x)}$. Чтобы убедиться в том, что при этом последние векторные поля симплектически ортогональны между собой, нам необходимо дополнительное исследование условия $(*)$.

Пусть условие $(*)$ выполнено для произвольной гладкой функции $h$ на $Y$. Тогда если гамильтоново векторное поле $X_{h}$ не обращается в нуль в некоторой окрестности точки 
$p \in Y$, то из условия $(*)$ следует, что существует не обращающаяся в нуль вещественная функция

$$
\alpha_{h}: X \backslash(B \cup \text { Sing }) \rightarrow \mathbb{R}, \quad \text { так, что } \quad X_{\psi^{*} h}=\alpha_{h} \nabla_{\psi} X_{h} .
$$

Тогда нетрудно видеть, что функция $\alpha$ должна быть одна и та же для всех функций. В самом деле, применим условие $(*)$ к произведению пары $h_{1} \cdot h_{2}$. Тогда получаем

$$
\nabla_{\psi} X_{h_{1} \cdot h_{2}} \wedge X_{\psi^{*}\left(h_{1} \cdot h_{2}\right)}=\psi^{*} h_{1} \cdot \psi^{*} h_{2}\left(\alpha_{h_{1}} \nabla_{\psi} X_{h_{1}} \wedge \nabla_{\psi} X_{h_{2}}+\alpha_{h_{2}} \nabla_{\psi} X_{h_{2}} \wedge \nabla_{\psi} X_{h_{1}}\right),
$$

откуда видно, что условие $(*)$ выполнено тогда и только тогда, когда $\alpha_{h_{1}}=\alpha_{h_{2}}$ для любой пары функций, откуда следует универсальность $\alpha$.

Далее, в слое $\psi^{-1}(p)=Q_{p} \backslash B$ над $p$ рассмотрим в произвольной точке $x \in \psi^{-1}(p) \subset X$ разложение касательного пространства $T_{x} X=T_{x}^{\mathrm{hor}} \oplus T_{x}^{\mathrm{vert}}$, задаваемое симплектической связностью $\nabla_{\psi}$, и возьмем ограничение симплектической формы $\omega_{X}$ на горизонтальную компоненту, обозначив его $\omega_{X}^{\text {hor }}$. Тогда нетрудно видеть, что геометрический смысл функции $\alpha$ таков: для любой пары векторов $v_{1}, v_{2} \in T_{x}^{\text {hor }}$ имеем равенство

$$
\omega_{X}^{\text {hor }}=\alpha(x) \omega_{Y}\left(d \psi\left(v_{1}\right), d \psi\left(v_{2}\right)\right) .
$$

Отсюда следует, что если два гамильтоновых векторных поля были симплектически ортогональны внизу на $Y$, то их подъемы посредством $\nabla_{\psi}$ наверх останутся симплектически отрогональными. Применяя снова условие $(*)$, получаем, что $\psi^{*} h_{j}$ коммутируют в $X$. Но построенный нами тор $T(x)$ есть совместное множество уровней функций $f_{1}, \ldots, f_{k}, \psi^{*} h_{1}$, $\ldots, \psi^{*} h_{n-k}$, определенных в его окрестности и коммутирующих между собой. Отсюда следует, что $T(x)$ - лагранжев.

ЗАмечАниЕ. На самом деле для построения подъема лагранжева тора из $Y$ в $X$ мы нигде не пользовались тем, что $Y$ есть торическое многообразие. Поэтому конструкция такого рода подъема может быть использована в общей ситуации, когда на компактном симплектическом многообразии действует вещественный тор неполной размерности.

Доказанная выше теорема может быть использована для построения гамильтоново неизотопных монотонных лагранжевых торов в произвольных монотонных симплектических многообразиях (например, в многообразиях Фано). Возвращаясь к примеру, приведенному выше, заметим, что подъемом лагранжевых торов можно получить соответствие

$$
H_{1}\left(Y \backslash D_{\text {Sing }}, \mathbb{Z}\right) \rightarrow \text { классы лагранжевых торов, }
$$

при этом нетривиальным это соответствие в примере становится только для монотонных торов. В этом случае ожидается, что каждый из трех примитивных классов из

$$
H_{1}\left(Y \backslash D_{\text {Sing }}, \mathbb{Z}\right)=H_{1}\left(\mathbb{C P}^{1} \backslash \text { три точки, } \mathbb{Z}\right)
$$

порождает свой класс монотонного лагранжева тора в многообразии Фано $F^{3}$ (см. пример выше), и эти три класса попарно гамильтоново неизотопны.

\section{СПИСОК ЦИТИРОВАННОЙ ЛИТЕРАТУРЫ}

[1] С. А. Белев, Н. А. Тюрин, Матем. заметки, 87:1 (2010), 48-59. [2] Н. А. Тюрин, $T M \Phi, \mathbf{1 6 2 : 3 ~ ( 2 0 1 0 ) , ~ 3 0 7 - 3 3 3 . ~ [ 3 ] ~ Н . ~ А . ~ Т ю р и н , ~ Т М Ф , ~ 1 6 7 : 2 ~ ( 2 0 1 1 ) , ~ 1 9 3 - 2 0 5 . ~ [ 4 ] ~ A . ~ W e i n s t e i n , ~}$ Ann. of Math. (2), 98 (1973), 377-410.

\section{С. А. Белёв}

Поступило

Объединенный институт ядерных

09.11.2011

исследований, г. Дубна Московской обл.

\section{Н. А. Тюрин}

Объединенный институт ядерных

исследований, г. Дубна Московской обл.

E-mail: ntyurin@theor.jinr.ru 